\title{
Sequential Bifurcations in Sheared Annular Electroconvection
}

\author{
Zahir A. Daya, ${ }^{1,2}$ V.B. Deyirmenjian, ${ }^{1}$ and Stephen W. Morris, ${ }^{1}$ \\ ${ }^{1}$ Department of Physics, University of Toronto, 60 St. George St., Toronto, Ontario, Canada M5S $1 A 7$ \\ ${ }^{2}$ Center for Nonlinear Studies, MS-B258, Los Alamos National Laboratory, Los Alamos, NM 87545
}

(November 7, 2018)

\begin{abstract}
A sequence of bifurcations is studied in a one-dimensional pattern forming system subject to the variation of two experimental control parameters: a dimensionless electrical forcing number $\mathcal{R}$ and a shear Reynolds number Re. The pattern is an azimuthally periodic array of traveling vortices with integer mode number $m$. Varying $\mathcal{R}$ and Re permits the passage through several codimension-two points. We find that the coefficients of the nonlinear terms in a generic Landau equation for the primary bifurcation are discontinuous at the codimension-two points. Further, we map the stability boundaries in the space of the two parameters by studying the subcritical secondary bifurcations in which $m \rightarrow m+1$ when $\mathcal{R}$ is increased at constant Re.
\end{abstract}

In typical nonlinear pattern-forming systems, there is one easily adjusted quantity, the control parameter, whose variation results in a symmetry-breaking bifurcation to an ordered pattern [1]. If a second control parameter is available, then it may be possible to tune the parameters to codimension-two points at which two modes with different symmetries are equally unstable. Near such points, neighboring unstable modes compete with one another leading to low-lying secondary bifurcations even in the weakly nonlinear regime which is accessible to perturbative theoretical treatment. There are few experimental systems whose patterns can be conveniently manipulated by the independent variation of two control parameters. Examples include Rayleigh-Bénard convection $(\mathrm{RBC})$ in a rotating cylinder [2,3], convection in binary fluid mixtures [1, 4, and Taylor Vortex Flow (TVF) with rotation of both the inner and outer cylinders [1,5]. In most cases, the eventual two-dimensionality of the patterns results in a very complex phenomenology.

In this Letter, we study experimentally the bifurcations in a thin annular fluid film. The two-dimensional (2D) film is both sheared and driven into electroconvection [6]. This system is unique in that it involves two easily adjusted control parameters, the applied voltage and the shear, with the bifurcations to uni-directionally traveling vortices only in one-dimension (1D). The annular geometry makes the 1D pattern effectively periodic and free of boundary effects. Most other pattern-forming systems are fluid-mechanically three-dimensional (3D) and the competing patterns often have different symmetries and dimensions. For example, in TVF there is a codimension-two point where Taylor and wavy vortices are simultaneously unstable. The former (latter) pattern is $1 \mathrm{D}(2 \mathrm{D})$. The greatly increased size of the parameter space made available by the third dimension makes it more difficult to examine experimentally the instabilities to and between the competing pattern states. Further, it is important to understand which physical processes depend on the different symmetries of the competing modes and which on their different dimensions. In our system, symmetry alone determines the nature of the bifurcations.
The highly restricted geometry of our fluid film only allows the competition of azimuthally periodic $1 \mathrm{D}$ modes of various $m$-fold symmetries. As a function of voltage and shear, we find a simple sequence of primary and secondary bifurcations. The primary bifurcation is the transition from the conducting state to the convecting state, whereas the secondary bifurcations connect one convecting state to another. This scenario can be modelled by coupled Landau amplitude equations which can be calculated completely by perturbative methods from the primitive electrohydrodynamic equations [7]:8]. The amplitude equations reduce to a single generic Landau equation when applied to the primary bifurcation. We experimentally demonstrate that the coefficients of the nonlinear terms in this equation vary discontinuously at codimension-two points. Our experiments also reveal that the secondary bifurcations near codimensiontwo points are subcritical and suppressed by the shear.

The experimental system consists of an annular, freely suspended film of smectic A liquid crystal. The layered smectic A phase is isotropic and newtonian for flows in the plane of the film, and maintains a uniform thickness even while flowing. Such a film is driven to electroconvect by a voltage applied between the inner and outer edges which sets up an unstable distribution of surface charge on the film's free surfaces 9. A Couette shear is applied by mechanical rotation of the inner electrode. Previous studies of this instability explored the behavior of the primary bifurcation to electroconvection [6,9], confirming that the weakly nonlinear regime of convection near onset is described by a complex amplitude equation of the Landau form for a single mode number $m$ [10]. The concentric electrodes have inner (outer) radii $r_{i}\left(r_{o}\right)$, from which we define the radius ratio $\alpha=r_{i} / r_{o}$. The film forms an annular sheet of width $d=r_{o}-r_{i} \sim 1 \mathrm{~mm}$ and thickness $s \sim 0.1 \mu \mathrm{m}$ so that $s / d \sim 10^{-4}$. The fluid is treated as incompressible, strictly two-dimensional, and of uniform electrical conductivity. We denote the fluid density, molecular viscosity, and conductivity by $\rho, \eta$, and $\sigma$. In a typical experiment, a steady circular Couette shear was imposed by rotating the inner electrode at $\omega \mathrm{rad} / \mathrm{s}$ and a dc voltage $V$ was applied between the in- 
ner and outer electrodes. Current-voltage characteristics were obtained for incremental and decremental voltages. The state of the film is specified by the radius ratio and three additional dimensionless parameters. The control parameter $\mathcal{R}=\epsilon_{0}^{2} V^{2} / \sigma \eta s^{2}$, where $\epsilon_{0}$ is the permittivity of free space, is a measure of the external driving force. The Prandtl-like parameter $\mathcal{P}=\epsilon_{0} \eta / \rho \sigma s d$ is the ratio of the time scales of electrical and viscous dissipation processes in the film. The Reynolds number $\operatorname{Re}=\rho \omega r_{i} d / \eta$ indicates the strength of the applied shear and is the second control parameter. Details of the experimental apparatus and procedure can be found in Refs. [6, 9 11.

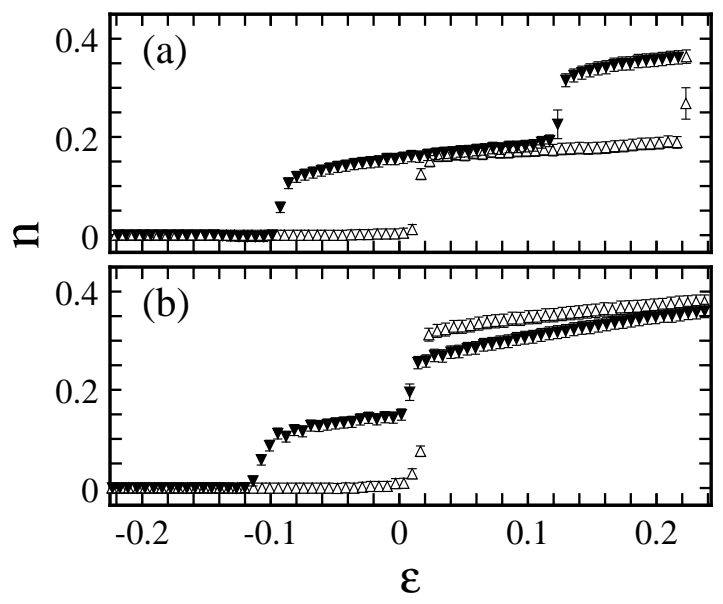

FIG. 1. Plots of $n$ versus $\epsilon$ for $\alpha=0.47$. In (a) $\mathcal{P}=15.3$, $\operatorname{Re}=0.94$. In (b) $\mathcal{P}=21.7, \operatorname{Re}=0.58$.

The primary bifurcation leads to a pattern of $m$ vortex pairs. Onset occurs at a critical voltage $V=V_{c}$ with the critical mode number $m=m_{c}$ becoming unstable. Linear stability predicts the values of $m_{c}$ and the critical control parameter $\mathcal{R}_{c} \propto V_{c}^{2}$ at which the film becomes marginally unstable. In general, $\mathcal{R}_{c}$ and $m_{c}$ are functions of $\alpha, \mathcal{P}$, and Re. However, when $\mathrm{Re}=0, \mathcal{R}_{c}=\mathcal{R}_{c}^{0}$ and $m_{c}=m_{c}^{0}$ are independent of $\mathcal{P}$. When $\operatorname{Re}>0$, it is found that $\mathcal{R}_{c}>\mathcal{R}_{c}^{0}$, for any $\alpha$ and $\mathcal{P}$, i.e. that the shear always acts to stabilize the conduction state and suppresses the onset of convection. In addition for nonzero shear, $m_{c} \leq m_{c}^{0}$. Both these predictions of linear theory have been experimentally confirmed. Our main experimental tool is the total electric current $I$, which is a measure of the rate of charge transport due to both conduction and convection. The reduced Nusselt number $n$ is the ratio of the current due to electroconvection to that due to conduction and is defined as $n=\left(I / I_{\text {cond }}\right)-1=(I / c V)-1$. The conductance of the film $c$ is obtained from the currentvoltage characteristic of the film when it is not convecting. Similarly, the reduced control parameter $\epsilon$, is given by $\epsilon=\left(\mathcal{R} / \mathcal{R}_{c}\right)-1=\left(V / V_{c}\right)^{2}-1$. The raw $(V, I)$ data can thus be transformed to measurements of $(\epsilon, n)$ [12]. In these dimensionless terms, the primary bifurcation occurs at $\epsilon=0$. When $\epsilon$ is increased, the secondary bifurca- tions are transitions between flows with mode numbers $m \rightarrow m+1$ 13. Upon decreasing $\epsilon$, the transitions $m \rightarrow m-1$ are observed. The mode changes can be observed directly, but rather crudely, using visual observations of slightly nonuniform films, or films seeded with small particles. They appear very cleanly, if more indirectly, in the form of jumps in $n$ as a function of $\epsilon$. These observations are in qualitative agreement with an equivariant bifurcation theory [14]. Increasing (decreasing) $\epsilon$ results in secondary bifurcations at $\epsilon_{1}<\epsilon_{2}<\ldots$ $\left(\epsilon_{-1}<\epsilon_{-2}<\ldots\right)$ corresponding to mode transitions $m \rightarrow m+1 \rightarrow \ldots(\ldots \rightarrow m+1 \rightarrow m)$.

In Figure 1 are illustrated two representative examples of secondary bifurcations. Figure $\mathbb{1}$ (a) shows $(\epsilon, n)$ data for a sheared film with subcritical primary and secondary bifurcations. The secondary bifurcation, which results in one additional traveling vortex pair, appears at $\epsilon_{1} \sim 0.22$ for increasing $\epsilon$. When $\epsilon$ is decreased, the removal of the vortex pair occurs at $\epsilon_{-1} \sim 0.12$. Convection altogether ceases at $\epsilon \sim-0.1$. Figure 11(b) shows a case where, for increasing $\epsilon$, the primary and secondary bifurcations appear as a single strongly subcritical bifurcation at $\epsilon \sim 0$. However, for decreasing $\epsilon, \epsilon_{-1}$ is distinct from $\epsilon \sim-0.11$ where convection stops. That shear selects the mode which is linearly unstable is well established in the case of the primary bifurcation [9]. It has been shown from linear stability analysis that the marginally unstable mode decreases from $m_{c}^{0} \rightarrow m_{c}^{0}-1 \rightarrow m_{c}^{0}-2 \ldots$ as Re is increased. Hence, for fixed $\alpha$ and $\mathcal{P}$, there are codimension-two points at particular Reynolds numbers, $\operatorname{Re}_{m, m-1}$, at which both $m$ and $m-1$ are marginally unstable [16]. For $\operatorname{Re}<\operatorname{Re}_{m, m-1}$, the primary bifurcation is to a convecting state with mode number $m$. The first secondary bifurcation occurs at $\epsilon=\epsilon_{1}>0$ to mode number $m+1$. For $\operatorname{Re}>\operatorname{Re}_{m, m-1}$, the primary bifurcation is to mode $m-1$. The first secondary bifurcation is at $\epsilon=\epsilon_{1}>0$ to mode $m$. Clearly, it follows that as $\mathrm{Re} \rightarrow \operatorname{Re}_{m, m-1}$ from above, $\epsilon_{1} \rightarrow 0$, resulting in the situation depicted in Fig. 1 (b). Our experimental protocol was always to hold Re constant and vary $\epsilon$. Neither experiment nor theory has addressed the question of what mode transitions occur if Re is varied for constant $\epsilon$.

Figures 2(a)-(h) show a sequence of $(\epsilon, n)$ data plots for $\alpha=0.56, \mathcal{P}=76$, and a range of closely spaced values of Re. In Fig. 2(a), the data at $\mathrm{Re}=0.124$ shows a subcritical primary bifurcation with mode number $m=8$. For the range of $\epsilon$ investigated, secondary bifurcations were not encountered. In Fig. 2(b), the data at $\mathrm{Re}=0.142$ shows a subcritical primary bifurcation with $m=7$ followed by a subcritical secondary bifurcation $(m=7 \rightarrow 8)$ at $\epsilon_{1} \sim 0.26$. Hence at some Reynolds number, $0.124<\operatorname{Re}_{8,7}<0.142$, there is nascent at $\epsilon=0$ the mode change $8 \rightarrow 7$. At $\operatorname{Re}=0.160$, the secondary bifurcation is suppressed to higher $\epsilon_{1} \sim 0.48$, as shown in Fig. 2(c). The sequence of events in Figs. 2(a) through (c) repeats with increasing Re. In Fig. 2(f), data at $\operatorname{Re}=0.214$ shows a primary bifurcation to $m=6$ and a secondary bifurcation at $\epsilon_{1} \sim 0.07$ to $m=7$. Hence a 
second codimension-two point at $0.196<\mathrm{Re}_{7,6}<0.214$ is traversed. At $\operatorname{Re}=0.231$ and $\operatorname{Re}=0.249$, the secondary bifurcation is suppressed to $\epsilon_{1} \sim 0.11$ and $\epsilon_{1} \sim 0.16$ respectively, as is shown in Figs. 2(g) and (h). The data obtained while $\epsilon$ is being reduced has equally interesting behavior. For example, for increasing $\epsilon$ in Fig. 2(f) there is a $m=6 \rightarrow 7$ bifurcation, but for decreasing $\epsilon$ the $m=7$ convecting state exits directly to conduction by a single discontinuous transition, without recourse to the $m=6$ state. However, for the slightly higher Re in Figs. 2(g) and (h), the $m=6$ state is revisited while $\epsilon$ decreases.

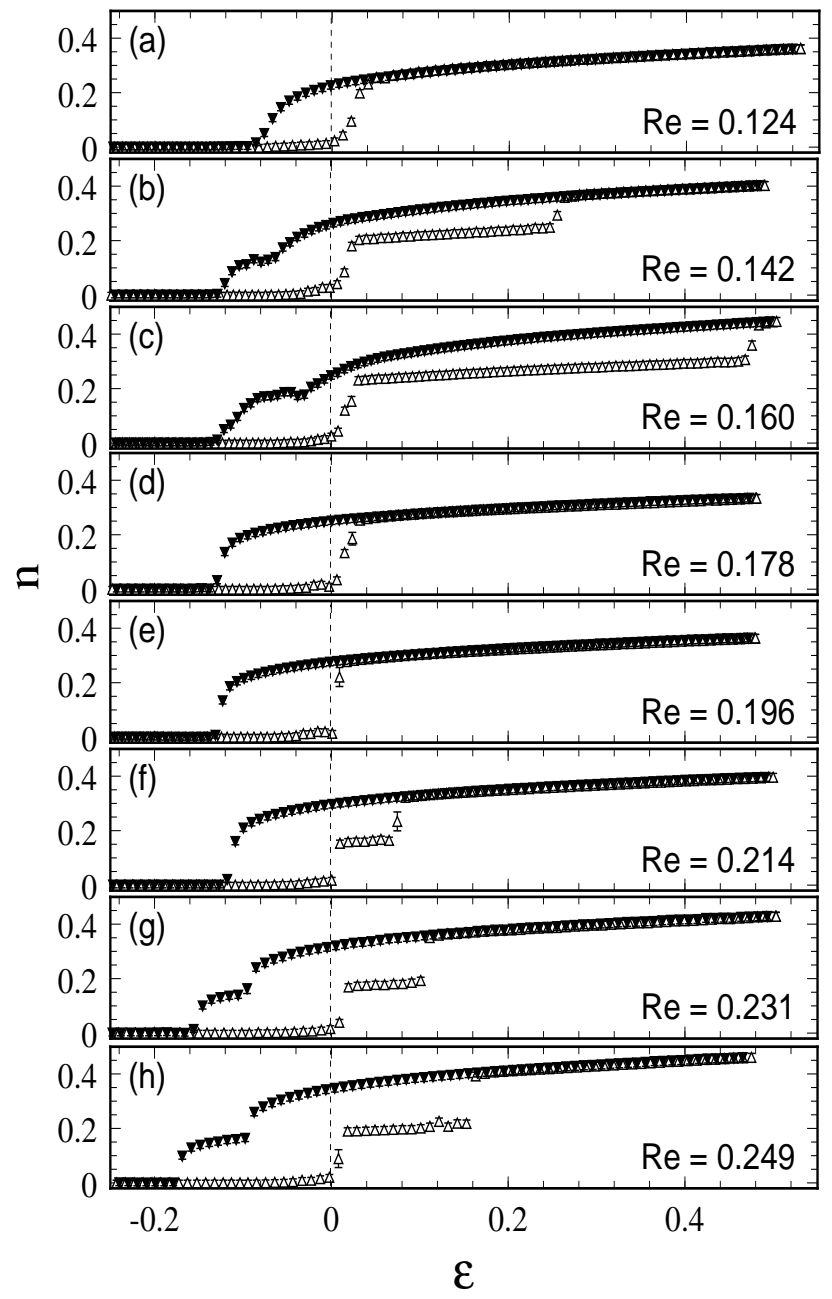

FIG. 2. A Re-sequence demonstrating mode changing bifurcations. Plotted are $n$ versus $\epsilon$ at $\alpha=0.56$ and $\mathcal{P}=76$ for a sequence of increasing Re. The open (filled) triangles are for increasing (decreasing) $\epsilon$ data.

For nonzero shear, symmetry and perturbative analyses of the primitive equations lead to the amplitude equation

$$
\begin{array}{r}
\tau\left(\dot{A}_{m}-i a_{I m} A_{m}\right)=\epsilon\left(1+i c_{0 m}\right) A_{m} \\
-g_{0 m}\left(1+i c_{2 m}\right)\left|A_{m}\right|^{2} A_{m}-g_{1 m}\left(1+i c_{3 m}\right)\left|A_{n}\right|^{2} A_{m}
\end{array}
$$

$$
-h_{0 m}\left(1+i c_{4 m}\right)\left|A_{m}\right|^{4} A_{m}+\ldots,
$$

and its counterpart under $m \leftrightarrow n$ symmetry [8]. In the above, all of the coefficients are real. The steady state solutions of the real part of Eq. 0.1 are consistent with the experimental observations of a state $m$ being replaced by a state $n=m \pm 1$ via a discontinuous transition [8]. To further interpret the data in Fig. 2 and better understand the behavior of the primary bifurcation as the Reynolds number is increased through $\operatorname{Re}_{8,7}$ and $\operatorname{Re}_{7,6}$, we consider the real and steady state part

$$
\epsilon A-g A^{3}-h A^{5}=0,
$$

of Eq. 0.1, where $A$ is real, $g \equiv g_{0 m}$, and $h \equiv h_{0 m}$. We have truncated at quintic order in the expansion. Since the reduced Nusselt number $n=A^{2}$ [7, 10], we can determine $g$ and $h$ for each primary bifurcation in the sequence in Fig. 2. Our results are plotted in Fig. 3. We find that both $g$ and $h$ are strongly discontinuous as Re is increased through the codimension-two points $\operatorname{Re}_{8,7}$ and $\operatorname{Re}_{7,6}$. The discontinuity in both $g$ and $h$ is smaller at $\operatorname{Re}_{8,7}$ than at $\mathrm{Re}_{7,6}$. This raises the possibility that these discontinuities may vanish in the limit $m \rightarrow \infty$ and so are a feature of the finite size and discrete mode structure of this system.

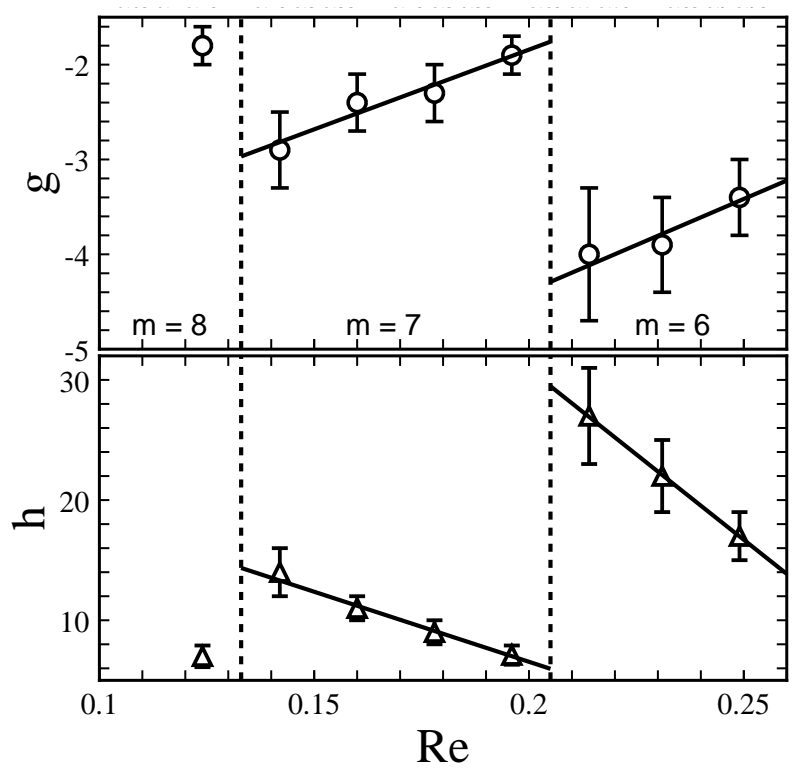

FIG. 3. The coefficients $g$ and $h$ in Eq. 0.2 versus Re for the data in Fig. 2. The dashed lines locate the codimension-two points. Solid lines are linear fits to the data.

A minimal description of a state consists of identifying the value of its mode number $m$. Allowing for hysteresis, each state persists over certain overlapping ranges of $\alpha$, $\mathcal{P}, \mathcal{R}$, and Re. Here, we focus on fixed $\alpha$ and $\mathcal{P}$ for simplicity 15. In Fig. [4 we map the solutions in the subspace defined by $(\epsilon, \operatorname{Re}, \alpha=0.56, \mathcal{P}=76)$, where the reduced variable $\epsilon$ is used for convenience. Figure 7 shows the values of $\epsilon$ at which conduction and convection with 
various $m$ were found, for given values of Re. Below (above) the lowermost dashed lines (horizontal solid line), only conduction (convection) occurs. Between these lines both conduction and convection are observed, depending hysteretically on whether $\epsilon$ is increasing or decreasing. A tricritical point(TC) is shown at $\operatorname{Re} \sim 0.11$. For $\operatorname{Re} \lesssim$ 0.11 the onset of convection is supercritical and hence non-hysteretic [10].

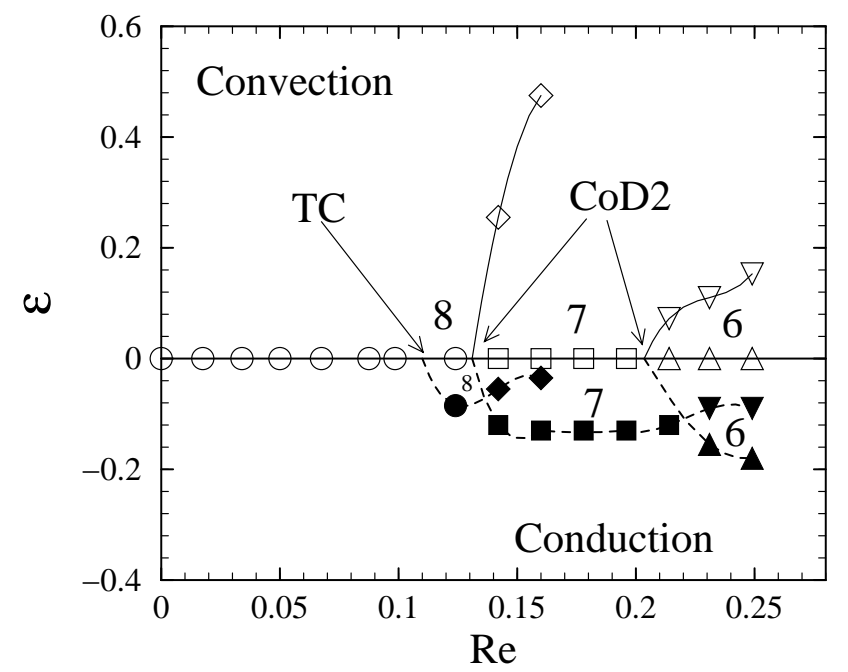

FIG. 4. Plot of the parameter space of patterns with mode numbers $m$ for $\alpha=0.56$ and $\mathcal{P}=76$. Open (closed) symbols indicate conduction $\rightarrow m(m \rightarrow$ conduction $)$ and $m \rightarrow m+1$ $(m+1 \rightarrow m)$ transitions encountered for increasing (decreasing) $\epsilon$. The numbers are the observed $m$. The region below (above) $\epsilon=0$ is multistable with conduction ( $m$ and $m+1$ states). TC is a tricritical point that connects the supercritical and subcritical branches. CoD2 are codimension-two points. The solid lines for $\epsilon>0$ are the secondary stability boundaries across which $m \rightarrow m+1$. Each of the dashed lines for $\epsilon<0$ connects the positions of the saddle-nodes for mode $m$.

Within the convecting regime, the boundaries of the secondary bifurcations between $m=8,7$ and $m=7,6$ show upwards trends indicating that an analogous suppression occurs for the modes $m=8$ and $m=7$, even after they cease to be the primary mode of bifurcation [17]. The suppression of the secondary modes increases more rapidly with Re than when they were the primary mode, with larger $m$ modes suppressed more rapidly than smaller $m$ modes. Inside the hysteresis loop of the primary bifurcation, we found as many as three possible distinct states, one being conduction. (See Figs. 2(b), $(\mathrm{c}),(\mathrm{g})$, and $(\mathrm{h}))$. The entire portrait presented in Fig. 1 depends perhaps strongly on $\alpha$ and weakly on $\mathcal{P}$ and is likely to be more branched (contain more transitions) for larger $\alpha$. It should be possible to generate theoretically such a tree from the primitive equations, once they have been reduced to the form of Eq. 0.1 [8].

We have shown that in our discrete-mode system, sec- ondary bifurcations appear near codimension-two points. Using a generic Landau model, we have quantitatively analyzed the behavior of the primary bifurcations on either side of these points. We have found that the coefficients of the cubic and quintic terms of the Landau equation are strongly discontinuous at the codimensiontwo points. We expect that this scenario occurs over large regions of our parameter space and may well be a general property of discrete-mode pattern-forming systems such as TVF. 河 Unlike TVF however, this system only has bifurcations between one-dimensional patterns with simple $m$-fold symmetries. The parameter space is characterized by branches that demarcate different stable and multistable domains. The surprizingly intricate bifurcation structure of this highly geometrically constrained instability is amenable to detailed quantitative analysis starting from the underlying electrohydrodynamic equations. [8] As such, it is an ideal venue for experimental and theoretical studies of bifurcations in finite nonlinear pattern-forming systems.

We thank R. E. Ecke for helpful discussions and constructive comments. This research was supported by the Canadian NSERC and the U.S. DOE (W-7405-ENG-36).

[1] M. C. Cross and P. C. Hohenberg, Rev. Mod. Phys. 65, 851 (1993).

[2] Y. Liu and R. E. Ecke, Phys. Rev. E 59, 4091, (1999).

[3] F. Zhong, R. E. Ecke, and V. Steinberg, Phys. Rev. Lett. 67, 2473 (1991).

[4] I. Rehberg and G. Ahlers, Phys. Rev. Lett. 55, 500, (1985).

[5] P. Chossat and G. Iooss, The Couette-Taylor Problem (Springer-Verlag, New York, 1994).

[6] Z. A. Daya, V. B. Deyirmenjian, S. W. Morris, and J. R. de Bruyn, Phys. Rev. Lett. 80964 (1998).

[7] V. B. Deyirmenjian, Z. A. Daya, and S. W. Morris, Phys. Rev. E 56, 1706 (1997).

[8] V. B. Deyirmenjian, Z. A. Daya, and S. W. Morris, to be published.

[9] Z. A. Daya, V. B. Deyirmenjian, and S. W. Morris, Phys. Fluids 11, 3613 (1999).

[10] Z. A. Daya, V. B. Deyirmenjian, and S. W. Morris, Phys. Rev. E 64, 036212 (2001).

[11] Z. A. Daya, Ph. D. Thesis, University of Toronto, 1999.

[12] $V_{c}$ is determined by fitting the $(\epsilon, n)$ data to the appropriate Landau amplitude equation. See Refs. [10] and [11].

[13] Mode transitions $m \rightarrow m \pm n$ with $n>1$ are unlikely except at large $\alpha$.

[14] W. F. Langford and D. D. Rusu, Physica A261, 188 (1998).

[15] $\mathcal{P}$ slowly varied by less than $2 \%$ 10,11 over the course of a run due to the electrochemical-drift of the electrical conductivity of the liquid crystal.

[16] It is also possible to reach codimension-two points by 
tuning $\alpha$ even for $\operatorname{Re}=0$. Codimension-three or -four points, though unlikely, are possible since $\epsilon, \operatorname{Re}, \alpha$, and $\mathcal{P}$ are all tunable.

[17] The suppression of the primary mode is set to zero by the definition of $\epsilon$. 